\title{
COMPARISION STUDY OF MICROBIAL GROWTH OF INJECTION MOLDED PEEK AND BRE. FLEX MATERIALS FOR BOUNDED REMOVABLE PARTIAL DENTURES (RANDOMIZED CLINICAL TRIAL)
}

\author{
Moataz Ibrahim Hashim*, Nadia A Abbas ** and Nora Cheta***
}

\begin{abstract}
This study aimed to: compare the adhesion of bacteria by using Brain heart infusion agar plates in class III modification I maxillary partially edentulous patients wearing removable partial dentures constructed from two different injectable thermoplastic materials PEEK and BRE.FLEX (second edition). The result of the study showed The PEEK group showed a greater bacterial growth than the Bre.Flex group in all the follow up periods and the difference was statistically significant.

Materials and methods: twenty-six patients having Kennedy class III modification I Maxillary partially edentulous ridges with fully dentate mandibular arch were divided into two equal groups to fabricate the thermoplastic materials. The first group received Bre.Flex material. The second group received PEEK material. The adhesion of bacteria from two groups were measured by using (brain heart infusion agar plate): at the time of denture insertion, after one week and after four weeks of denture insertion. The collected data were tabulated and statistically analyzed.
\end{abstract}

Results: showed that the difference between the two groups was statistically significant was reported between two groups regarding bacterial growth.

Conclusion: Within the Bre.Flex group, the bacterial growth has increased as the follow up period increased. Within the PEEK group, the bacterial count has increased as the follow up period increased. The PEEK group showed a greater bacterial growth than the Bre.Flex group in all the follow up periods and the difference was statistically significant.

KEYWORDS: PEEK, thermoplastic materials, bacterial growth

\footnotetext{
* MDS student, Department of Prosthodontics, faculty of Dentistry, Cairo University.

** Professor of Prosthodontic, faculty of Dentistry, Cairo University.

*** Assistant Professor of Prosthodontic, faculty of Dentistry, Cairo University
} 


\section{INTRODUCTION}

Removable partial denture is cost effective and reversible treatment method for partially edentulous patients at any age. With the changing trends in dental treatment that favor retention of natural teeth, a decline in the number of complete dentures with the increase in the number of removable partial dentures(RPDs)is anticipated. ${ }^{(\mathbf{1})}$.

A successful removable partial denture must meet important criteria as: function, comfort, esthetics, cost and built-in performance, in addition to easy cleanliness and ability to prevent the adhesion of microorganisms on its surface. The oral deposits and microorganisms that adhere to prosthesis lead to several undesirable effects. The adherent material itself is unaesthetic in appearance and unpleasant in terms of tactile sensation, taste, and odor. the masticatory requirement of an individual. This does not consider the subjective number of teeth perceived required for aesthetics, comfort and confidence. ${ }^{(2)}$.

Most removable partial dentures are used for the replacement of missing natural teeth are fabricated from metallic and/or acrylic components. Esthetics in the anterior region can be obtained by using a removable partial denture over a fixed restoration, especially when there is loss of soft/hard tissues surrounding the abutment teeth. If a metal clasp arm of the denture terminates in the undercut of a tooth in an aesthetic zone area, this will result in poor esthetic. ${ }^{(3)}$ Thermoplastic materials as Breflex and PEEk have been used in removable partial denture construction; they are becoming a potential pathogenic factor for oral mucosa being in contact with this material. ${ }^{(4)}$

Polyamide resin was proposed as a denture base material in the 1950s. Chemically, the original nylon is a PA 12 (polyamide). Nylon is a generic name for certain types of thermoplastic polymers belonging to the class known as polyamides. Thermoplastic nylon is a polyamide resin derived from diamine and dibasic acid monomers. Nylon is a versatile material, suitable for a broad range of applications. ${ }^{(5,6)}$ PEEK is used in various dental applications mainly are: dental implants, implant abutments, fixed crowns, fixed bridges and removable dentures. ${ }^{(7)}$

The denture clasps made of PEEK have lower retentive forces compared to cobalt-chromium (Co-Cr) clasps. However, since the study was conducted on metal crowns in vitro, it is not known how effective the esthetic PEEK clasps would be in retaining dentures in the clinical setting. ${ }^{(8)}$

\section{MATERIALS AND METHODS}

Twenty-six patients were selected from the outpatient clinic of Prosthodontic department, Faculty of dentistry, Cairo University. The inclusion criteria of patients selection were All patients were having Kennedy class III modification I Maxillary partially edentulous ridges with fully dentate mandibular arch, all patients have skeletal maxillo mandibular relationship with sufficient interarch distance,Male and Female patients with age range (40-55) with good oral hygiene and low caries index the remaining teeth have good periodontal conditions with no signs of attrition or gingival recession and free from any temporomandibular joint disorder. The study was designed to be a parallel randomized controlled trial. In terms of internal validity, randomized clinical trials represent the most scientifically rough study design, when properly performed, as they are best able to control bias and serve as a gold standard of study designs for evaluating treatment efficacy and widely considered as highest level of confirmatory scientific evidence. After construction of metal framework patients were randomly assigned into two identical groups by using a special web site concerned with randomization process called research randomizer (www.randomizer.org/).

The patients were randomly assigned to either one of two groups; the first group: Patients received maxillary removable partial denture fabricated 
from Injectable Bre.Flex second edition (Bredent, $2^{\text {nd }}$, Germany) resin material reinforced by metal framework. While the second group: Patients received maxillary removable partial denturefabr icatedfrominjectablepolyrtheretherketone(PEEK) (GMbH\&Co.KG.Germany) reinforced by metal framework.

Preliminary impressions were made for the patient's maxillary and mandibular arches by using irreversible hydrocolloid (alginate) (Cavex CA37 Alginate impression material, Holland BV). Impressions were disinfected, then were poured in improved dental stone (Elite ${ }^{\circledR}$ rock dental stone, Zermack, Italy). Primary surveying was done on the diagnostic casts, and then face-bow record was used to mount the maxillary cast on semi adjustable articulator (A7 plus, Bio-Art Dental Products, São Carlos, SP, Brazil.). Diagnostic casts were mounted on a semi adjustable articulator to check for any teeth interferences. This was important to evaluate interarch distance to accommodate the future prosthesis. The mounted casts also were used to assess the antero-posterior jaw relation.

Panoramic and periapical radiographs were performed as a complete mouth survey to evaluated the bone index areas and crown root ratio. The selected patients were informed about the participation in scheduled follow-up for 1 month after receiving the removable partial denture by a written informed consent. An informed consent was signed by each patient as it is one of the most important facets of bioethics to make sure that a patient understands the risks and benefits of any medical procedure. (Ilfeld 2006) stated that Requiring informed consent protects many patients from being forced to participate in medical studies without understanding the risks involved.

Mouth preparation was made including guide lines preparation and rest seat preparation.
The maxillary final impression was taken using medium-bodied elastomeric impression material (AquasilMonophase, DENTSPLY CAULK, USA) mixed according to the manufacturer's instructions. Impression was boxed and poured, in extra hard dental stone (Elite ${ }^{\circledR}$ rock dental stone, Zermack, Italy). The master cast was modified by drawing the design which is palatal strap major connector, Aker's clasps were drawn on the molars and Aker's arm was drawn on the canine then duplicated into a refractory cast by using silicon (Technosil, Bredent, Germany) to fabricate the metal framework. The use of Removable partial denture made from combination of thermoplastic resin and metal is now rapidly gaining popularity among general dentists and is considered to be superior to conventional metal-clasp retained RPDs with metal clasps in terms of both esthetics and comfort as the rigidity of the metallic framework distribute the forces equally and thermoplastic clasps enhance the esthetic so these type of RPD made a combination of esthetic and mechanical point of view.

The Bre.flex resin was preheated at 222 Co for 15 minutes by using the injection molding unit (Thermoflex 400), then Heated softened bre.flex resin was injected into the mold in 90sec After curing, the dentures were deflasked then; they were ready for finishing and polishing then Each denture was finished and polished using thermal resin finishing burs at low speed, pumice, and finally buffing with swans down mop was done to add a very high luster.

While Polyetheretherketone PEEK was preheated at 400 Co for 20 minutes by using the injection molding unit (Thermoflex 400) then Heated softened PEEK was injected into the mold by pressure 950mega pascal and velocity 6 bars in 240 seconds. After curing, the dentures were deflasked then; they were readyfor finishing and polishing. 


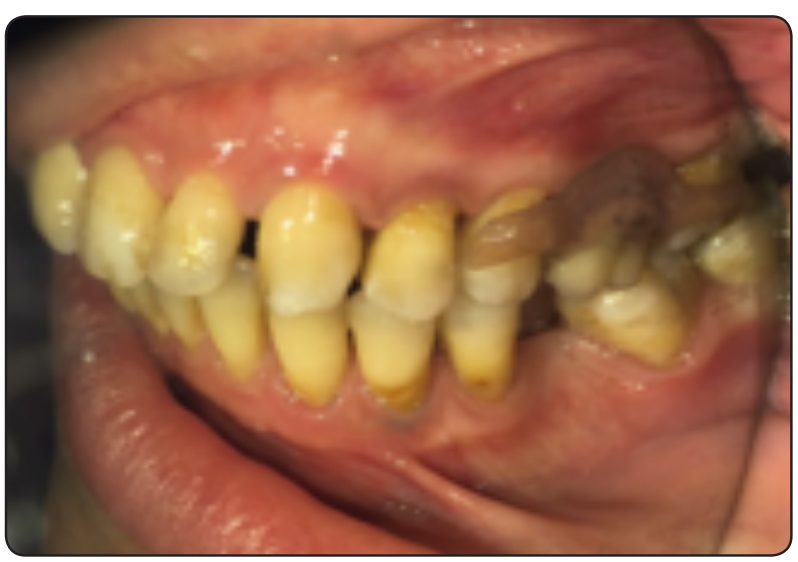

Fig. (1) Bre_flex clasp

\section{Microbiological Evaluation.}

After the denture was delivered the bacterial growth was recorded immediately after insertion, first week and fourth week following insertion using the brain heart infusion agar plate

\section{Swab collection}

Swab was collected from an area of $(1 \mathrm{~cm} \mathrm{x} 1 \mathrm{~cm})$ dimension in the internal surface of the maxillary partial denture and from oral mucosa covering the crest of ridge using sterile cotton swab.

\section{Preparation of culturing media}

Brain heart infusion agar plates were prepared by suspending $52 \mathrm{gm}$ of powder in $1000 \mathrm{ml}$ of distilled water then, heated to boiling to dissolve the medium completely. After that, it was allowed to cool to 45 $-50^{\circ} \mathrm{C}$ and poured in sterile petri plates.

\section{Cultivation of bacteria}

1. Swabs were emulsified in $1 \mathrm{ml}$ nutrient broth then three serial dilution (10-1, 10-2 10-3) were made for each sample. This done by adding 0.1 $\mathrm{ml}$ of the sample to $0.9 \mathrm{ml}$ sterile broth to make dilution of 1:10 .The previous step was repeated to reach dilution of $1: 100$ and then dilution of 1:1000.

2 . The resulting samples were immediately plated in Brain Heart Infusion agar to determine the total number of microorganisms.

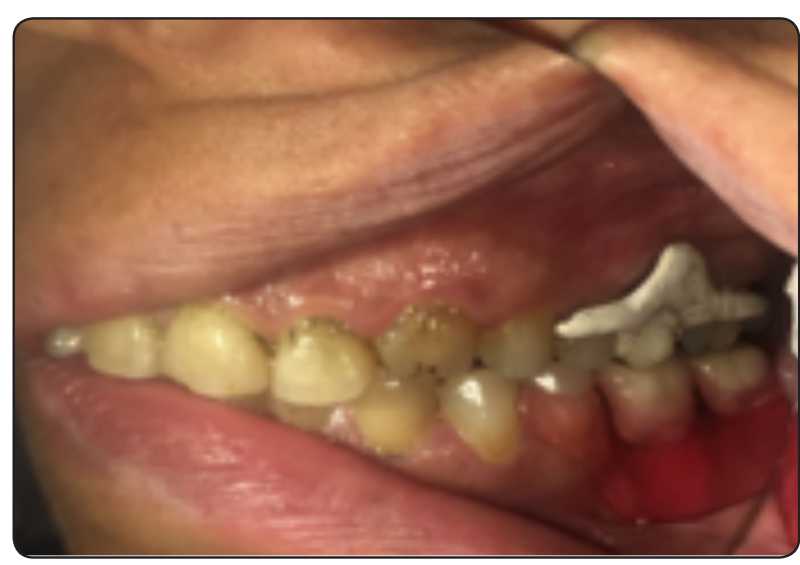

Fig. (2) PEEK clasp

3. Three dishes were covered and incubated at $37^{\circ} \mathrm{C}$ for 24 hours under aerobic conditions and another three plates were incubated at $37^{\circ} \mathrm{C}$ for 48 hours under anaerobic conditions.

\section{Estimation of bacterial number}

Viable colonies on each petri dish were counted visually and the estimated number of colony forming units (CFU) per milliliter was calculated

$\mathrm{CFU}=$ Total number of colonies counted in the plate $\mathrm{x}$ inversion of saline dilution $\mathrm{x}$ inversion of the cultured volume therefore:

CUF $\mathrm{ml}=$ Total number of colonies counted in the plate $\mathrm{x}$ inversion of the culture volume $\mathrm{x} 1000$

The number of colonies that formed under aerobic and anaerobic conditions were recorded separately

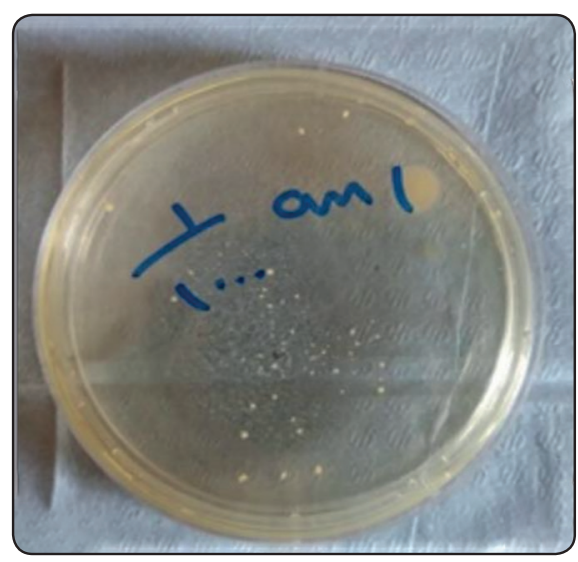

Fig (3) Bacterial colonies under anaerobic conditions 


\section{RESULTS}

Statistical analysis was performed with SPSS $20^{\circledR}$, Graph Pad Prism ${ }^{\circledR}$ and Microsoft Excel 2016.

All the colonies were counted in one sector and then multiplied by the number of sectors. $\mathrm{CFU} / \mathrm{ml}=$ total number of colonies counted in the plate X 50 .

All data were explored for normality by using Shapiro Wilk Normality test which revealed that data follow normal distribution accordingly Independent t-test was used for comparisons.

Data from the two groups were collected, tabulated and statistically analyzed and illustrated in tables and graphs. The mean and standard deviation values were calculated for two group. Paired sample t-test was used to test the difference between two groups in related samples.

\section{Mucosal surface}

At Baseline (T0) The calculated means of colonies forming unit $(\mathrm{CFU} / \mathrm{ml})$ and standard deviations from the mucosal surface in Bre flex group at baseline (T0) was $(83.33 \pm 8.1)$ and (15.6 \pm 4.3$)$, while in PEEK group it was $(150 \pm 48.3)$ and $(41.8 \pm 16.2)$ for aerobic and anaerobic bacteria respectively.

Comparison between Bre flex group and PEEK group showed significant difference as $\mathbf{p}$-value $<0.05$.

After 1 week (T1) The calculated means of colonies forming unit $(\mathrm{CFU} / \mathrm{ml})$ and standard deviations from the mucosal surface in Bre flex group after 1 week (T1) was $(57.4 \pm 11.4)$ and $(17.5 \pm$ $6.01)$, while in PEEK group it was (106.6 \pm 44.7$)$ and $(27.4 \pm 8.6)$ for aerobic and anaerobic bacteria respectively

Comparison between Bre flex group and PEEK group after 1 week (T1) showed significant difference as $\mathbf{p}<0.05$.

Statistical Package for Social Science, IBM, USA.

Graph Pad Technologies, USA.

Microsoft Co-operation, USA.
After 4 weeks ( $\begin{aligned} & \text { T 2) The calculated means } \\ & \text { 2 }\end{aligned}$ of colonies forming unit $(\mathrm{CFU} / \mathrm{ml})$ and standard deviations from the mucosal surface in Bre flex group after 4 weeks (T2) was $(52.5 \pm 20.5)$ and $(16.7 \pm 5.6)$, while in PEEK group it was (135.4 $\pm 33.7)$ and $(58 \pm 19.3)$ for aerobic and anaerobic bacteria respectively showed significant difference as $\mathbf{p}<0.05$.

\section{DENTURE SURFACE}

At Baseline (T0) The calculated means of colonies forming unit $(\mathrm{CFU} / \mathrm{ml})$ and standard deviations from the denture surface at baseline (T0) was (0) for Bre_flex and PEEK regarding aerobic \& anaerobic bacteria

Results showed that there is insignificant difference between Bre-flex\& PEEK regarding aerobic \& anaerobic also between aerobic \& anaerobic within the same group as $\mathrm{p}>0.05$

After 1 week The calculated means of colonies forming unit (CFU/ml) and standard deviations from the denture surface in Bre_flex group after 1 week (T1) was (29.2 \pm 8.9$)$ and (11.2 \pm 3.8$)$, while in PEEK it was $(69.5 \pm 25.3)$ and $(20.5 \pm 6.5)$ for aerobic $\&$ anaerobic bacteria respectively

Comparison between Bre_flex group and PEEK group at base line (T1) showed significant difference as $p<0.05$.

After Four Weeks (T2) The calculated means of colonies forming unit $(\mathrm{CFU} / \mathrm{ml})$ and standard deviations from the denture surface in Bre flex group after 4 weeks (T2) was $(37.4 \pm 9.6)$ and $(19.08 \pm 2.71)$, while in PEEK it was (109.4 \pm $20.8)$ and $(42.08 \pm 11.9)$ for aerobic \& anaerobic bacteria respectively. Comparison between Bre_flex group and PEEK group at base line (T2) showed significant difference as $p<0.05$. 
TABLE (1): Mean and standard Deviation of colony forming unit (CFU/ml) for Bre_flex and PEEK Group during follow up time:

\begin{tabular}{|c|c|c|c|c|c|c|}
\hline \multirow{2}{*}{\multicolumn{2}{|c|}{ Aerobic }} & \multicolumn{2}{|c|}{ Bre_flex } & \multicolumn{2}{|c|}{ PEEK } & \multirow{3}{*}{$\begin{array}{c}\text { P value } \\
<0.05^{*}\end{array}$} \\
\hline & & \multirow{2}{*}{$\begin{array}{c}\mathbf{M} \\
83.3\end{array}$} & \multirow{2}{*}{$\begin{array}{l}\text { SD } \\
8.1\end{array}$} & \multirow{2}{*}{$\begin{array}{c}\mathbf{M} \\
150\end{array}$} & \multirow{2}{*}{$\begin{array}{l}\text { SD } \\
48.3\end{array}$} & \\
\hline \multirow{3}{*}{ Oral mucosa } & T0 & & & & & \\
\hline & T1 & 57.4 & 11.4 & 106.6 & 44.7 & $<0.05^{*}$ \\
\hline & $\mathbf{T} 2$ & 52.5 & 20.5 & 135.4 & 33.7 & $<0.05^{*}$ \\
\hline \multirow{3}{*}{ Denture surface } & To & 0 & 0 & 0 & 0 & $>0.05$ \\
\hline & T1 & 29.2 & 8.9 & 69.5 & 25.3 & $<0.05^{*}$ \\
\hline & $\mathbf{T} 2$ & 37.4 & 9.6 & 109.4 & 20.8 & $<0.05^{*}$ \\
\hline
\end{tabular}

\begin{tabular}{|c|c|c|c|c|c|c|}
\hline \multirow{2}{*}{\multicolumn{2}{|c|}{ Anerobic }} & \multicolumn{2}{|c|}{ Bre_flex } & \multicolumn{2}{|c|}{ PEEK } & \multirow{2}{*}{ P value } \\
\hline & & M & SD & $\mathbf{M}$ & SD & \\
\hline \multirow{3}{*}{ Oral mucosa } & T0 & 15.6 & 4.3 & 41.8 & 16.2 & $<0.05^{*}$ \\
\hline & T1 & 17.5 & 6.01 & 27.4 & 8.6 & $<0.05^{*}$ \\
\hline & $\mathbf{T} 2$ & 16.7 & 5.6 & 58 & 19.3 & $<0.05^{*}$ \\
\hline \multirow{3}{*}{ Denture surface } & T0 & 0 & 0 & 0 & 0 & $>0.05$ \\
\hline & T1 & 11.2 & 3.8 & 20.5 & 6.5 & $<0.05^{*}$ \\
\hline & $\mathbf{T} 2$ & 19.08 & 2.71 & 42.08 & 11.9 & $<0.05^{*}$ \\
\hline
\end{tabular}

\section{DISCUSSION}

This study aim to evaluate the bacterial growth on both denture base and mucosa of the Bre-flex and PEEK partial denture reinforced by metal framework, Swabs from the mucosal surface under the PEEK denture base show statistically significant increase in the mean colonies forming units(CFU) count than the mucosal surface under the Bre_flex denture base through all follow up periods, That agrees with Menaka et all study in $\mathbf{2 0 1 0}$, swabs from the fitting surfaces at the base line time T0 ( time of insertions ) showed statistically no significant difference in the mean colonies forming units(CFU) count between Bre_flex and PEEK denture base as in $\mathrm{T} 0$ the aerobic and anerobic bacteria count were zero as the dentures didn't remain in patients mouth for long time during insertion. When comparing the result at first week and $4^{\text {th }}$ week follow up periods, PEEK recorded statistically significant higher bacterial count ${ }^{(9)}$. which agreed with Periera-cenici et all 2008 that found the microbial cells have been shown capability to adhere and colonize on both oral mucosa and denture base. ${ }^{(10)}$

Comparing the bacterial count within the same denture base material during the follow up time we found that, Aerobic bacteria count in oral mucosa swabs within the Bre_flex denture base material decrease, also in PEEK denture base material bacterial count decreased as aerobic bacteria is transient in oral cavity and it is not presented 
normally in oral cavity so it is normal to decrease in its count .

While the aerobic bacteria count from denture swabs within the same denture base material (Bre_ flex\&PEEK) increased during the follow up periods that may be due to the patients don't follow oral hygiene instructions.

On the other hand, anerobic bacteria count in oral mucosa swabs in Bre_flex denture base material group increased during follow up, also in PEEK oral mucosa swabs bacteria count increased during follow up. Also Anaerobic bacterial count in denture swabs within the same denture base material (Bre_flex\&PEEK) increased during the follow up periods that may be due to the patients don't follow oral hygiene instructions.

Insertion of any kind of restoration weather fixed or removable causes change in the oral microbial flora. The degree of change is varying according to material, type, and design of the restoration. It was proved that presence of dentures within the oral cavity may alter the nature of the microbial flora due to lack of cleansing effect by the tongue and saliva. Recent Studies confirmed aerobic and anaerobic microbial growth on the dentures and revealed that no two dentures had the same spectra of microorganisms. ${ }^{(10)}$

Microbial colonization to the Bre-flex denture base showed no difference between the colonization to the PEEK denture base in the early follow up periods but later microbial colonization in the PEEK denture base group showed higher numbers than Bre-flex denture base group.

The results of this study similar to Sargon Barkarmo et all who confirmed that the surface roughness had an impact on the bacterial adhesion to these materials, When comparing the effects of both material and time, The biofilm formation for S. sanguinis was significantly higher on PEEK and blasted PEEK compared with Ti6Al4V.also
S. oralis also grew to a higher extent on the blasted PEEK compared with all the other groups. It is known that increased surface roughness increases the amount of bacteria in the biofilm compared with a smoother surface (Teughels et al., 2006). One reason for this is that the bacteria can attach easier and become sheltered in the small micrometer scale cracks in the rougher surface (Bollen et al., 1997

The wettability of a biomaterial has also been proposed to influence the biofilm formation (Wassmann, Kreis, Behr, \& Buergers, 2017). Materials that have higher surface free energy will create a more wettable surface and are more likely to adhere bacteria (Teughels et al., 2006), although this depends on the hydrophobicity of the bacteria (Song, Koo, \& Ren, 2015)

Factors that affect microbial adhesion to denture base resin materials include surface free energy, topography, roughness of the surface and micro porosities, surface charge of the microorganisms and presence of saliva coating, all these factors can have explained why PEEK showed a higher statistical significance difference than Breflex as with time the surface roughness and micro porosities of PEEK increase so PEEK denture surface showed more bacterial count more than Breflex denture surface. ${ }^{(11)}$ (Al-Bakri et al., 2014)

The present results agreed with Sammar Mohamed, et al.2016 that found that the Bre-flex material better results and more favorable biological reaction than the acrylic resin dentures throughout the study period and less microbial colonization.

Regarding the changes that occurred by time between both groups revealed that the level of microbial colonization changed depending on the age of the prosthesis there was an increase in microbial colonization during late follow up periods. The number of microbes increased over time as the denture base material aged. Not only the age of prosthesis that controlled the increase of the microbial colonization on the oral cavity surface (mucosa and denture base). ${ }^{(12)}$ 


\section{CONCLUSION}

From this study, it could be concluded that:

1) Both the denture base materials fabricated from Bre_flex and PEEK have the affinity to support aerobic and anaerobic bacterial growth.

2) Bre_flex dentures have the less affinity to support aerobic and anaerobic bacterial growth comparing with PEEK dentures.

\section{REFRENCES}

1. Harvey W.L., Hoffman WM. Ten-year study of trends in removable prosthodontic service. J Prosthet Dent, 1989; 62:644-6646

2. Dewhirst FE, Chen T, Izard J, Paster BJ, Tanner AC, Yu WH, Lakshmanan A, Wade WG The human oral microbiome. J Bacteriol. (2010). 192:5002-5017.

3. Toole, G.O., Kaplan, H. B, \& KOlter, RBiofilm formation as Microbial Development. Annual Review of Microbiology. (2000). 49-79.

4. Ardelean L, Bortun C, Podariu A and Rusu L Manufacture of Different Types of Thermoplastic, Thermoplastic Composite Materials,INTECH, $1^{\text {st }}$ ed, Timisoara . Open Access Publisher(2012) 25-48
5. Vojdani $\mathbf{M}$ and Giti RPolyamide as a Denture Base Material : A Literature Review (2015). Journal of Shiraz University of Med Sci;16:1-9.

6. Ardelean L, Bortun C, Podariu A and Rusu L Manufacture of Different Types of Thermoplastic, Thermoplastic Composite Materials,INTECH, $1^{\text {st }}$ ed,Timisoara . Open Access Publisher(2012).25-48.

7. Najeeb S,Zafar MS,KhurshidZ and SiddiquiFApplications of polyetheretherketone (PEEK) in oral implantology and prosthodontics. J Prosthodont Res(2016); 60:12-19.

8. Tannous F, Steiner M, Shahin R and Kern M etentive forces and fatigue resistance of thermoplastic resin clasps. DentMat(2012) ;28:273-278.

9. Hamenaka, I., Iwamoto, m., Lassila, L.V.J., \&Vallittu, P. K. Wear resistance of injection molded thermpoplastic denture base resins Acta Biomater Odonto Scand. (2016) 2:31-37

10. Deepthi PV, Fernandez T, Karthikeyan S (2016). Oral microbiological flora in denture wearers - a review. J Adv Med Dent Sci Res. 4:92-94.

11. AL_AKhali, M., EL_Kerdawy, M., Ibraheim, Z., Abbas, N. \&Student. G. Compertative study on the microbial adhesion to acetal resin and metallic removable partial denture. (2012) Indian J Dent.3:1-4

12. Pereira-Cenci T, Del Bel Cury AA, Crielaard W and Ten Cate JM. Development of Candida-associated denture stomatitis: new insights. J Appl Oral Sci (2008); 16: 86-94. 